\title{
Off-resonance-robust velocity-selective magnetization preparation for non-contrast-enhanced peripheral MR angiography
}

\author{
Taehoon Shin ${ }^{1,{ }^{\star}}$, Bob S. Hu${ }^{1,2,3}$, and Dwight G. Nishimura ${ }^{1}$ \\ ${ }^{1}$ Department of Electrical Engineering, Stanford University, Stanford, California \\ ${ }^{2}$ Palo Alto Medical Foundation, Palo Alto, California \\ ${ }^{3}$ Heart Vista Inc., Palo Alto, California
}

\section{Abstract}

Purpose-To develop a new velocity-selective (VS) excitation pulse sequence which is robust to field inhomogeneity and demonstrate its application to non-contrast-enhanced (NCE) peripheral MR angiography (MRA).

\begin{abstract}
Methods-The off-resonance-robust VS saturation pulse is designed by incorporating $180^{\circ}$ refocusing pulses into the $\mathrm{k}$-space-based reference design and tailoring sequence parameters in a velocity region of interest. The VS saturation pulse is used as magnetization preparation for NCE peripheral MRA to suppress background tissues but not arterial blood based on their velocities. NCE peripheral MRA using the proposed VS preparation was tested in healthy volunteers and a patient with arterial stenosis.
\end{abstract}

Results-Calf angiograms obtained using the new VS preparation show more uniform background suppression than the reference VS preparation, as demonstrated by larger mean values and smaller standard deviations of artery-to-vein and artery-to-muscle contrast-to-noise ratios (71.0 \pm 11.4 and $75.3 \pm 12.1$ versus $61.7 \pm 22.7$ and $58.5 \pm 27.8$ ). Two-station peripheral MRA using the new VS preparation identifies stenosis of the femoral and popliteal arteries in the patient, as validated by digital subtraction angiography.

Conclusion-NCE MRA using the new VS magnetization preparation can reliably provide high angiographic contrast in the lower extremities with significantly improved immunity to field inhomogeneity.

\section{Keywords}

velocity-selective excitation; magnetization preparation; non-contrast-enhanced MRA; peripheral MRA

\section{Introduction}

Selective excitation of spin magnetizations based on their velocities can be a useful tool for generating image contrast in MRI. About two decades ago, a number of methods for velocity-selective (VS) excitation were demonstrated theoretically or experimentally to distinguish between flowing blood and stationary tissues for angiographic applications (15). These methods employed a sequence of a $90^{\circ}$ tip-down pulse, a bipolar gradient and a

*Correspondence to: Taehoon Shin, 350 Serra Mall, Packard 216, Stanford University, Stanford, CA 94305, Phone: (650) 724-3628, taehoons@stanford.edu. 
$90^{\circ}$ tip-up pulse (hereafter denoted by $90_{\mathrm{x}^{-}} G-90_{-\mathrm{x}}$ ) or its variants. The $90_{\mathrm{X}}-G-90_{-\mathrm{x}}$ module generates a sinusoidal excitation profile over velocity, yielding signal from moving materials with a varying strength depending on velocities, but no signal from stationary materials. However, the smooth transition between pass- and stop-bands in the sinusoidal velocity response hinders clear separation between arterial blood and background materials.

For direct acquisition of an artery-only image, a VS excitation profile should ideally have a pass-band and a stop-band in the expected velocity regions of arterial blood and background materials, respectively, with the narrowest possible transition-band. There were attempts to improve VS excitation toward an ideal excitation profile by a binomial expansion of the $\theta_{\mathbf{x}}$ $G$ - $\theta_{\mathrm{x}}$ sequence (6) or numerical optimization of similar expansions (7). These approaches lacked an explicit relationship between design parameters and a desired excitation profile, and therefore were not flexible in terms of achievable profile shapes.

The most systematic framework for designing VS excitation pulse sequences was provided by de Rochefort et al. (8). The excitation k-space initially introduced for spatially selective excitation by Pauly et al. (9), was extended to include the velocity Fourier variable. A VS excitation profile could be formulated as the Fourier transform of the $B_{1}$ field deposited in a generalized excitation k-space. Very recently, a VS inversion pulse sequence was designed based on the generalized excitation k-space formalism and the Shinnar-Le Roux (SLR) transform, and was successfully used as magnetization preparation for non-contrastenhanced (NCE) abdominal MR angiography (MRA) (10).

The k-space-based VS excitation has off-resonance sensitivity, which manifests as excitation profile shifting along velocity in proportion to off-resonance. In the previous study on VS inversion-prepared abdominal MRA, the effect of off-resonance-induced profile shifting turned out to be marginal due to relatively high systolic flow in the abdominal aorta and iliac arteries. However, in vasculatures which involve much lower arterial flow (e.g., the lower extremities), the excitation profile shifting may cause severe degradation of artery-tobackground contrast.

In this work, we present a new version of a VS excitation pulse sequence that significantly reduces the off-resonance sensitivity through two modifications. First, $180^{\circ}$ refocusing pulses are incorporated into the k-space-based sequence to zero out the phases created by off-resonance. Second, residual excitation errors due to imperfect $180^{\circ}$ rotations are further reduced in a finite velocity region of interest (ROI) by numerically tailoring sequence parameters. The performance of the proposed VS excitation pulse sequence is evaluated by numerical simulations and in vivo within a peripheral MRA protocol for imaging the lower extremities.

\section{Materials and Methods}

\section{Reference VS excitation}

Under the excitation k-space formalism, the transverse component of excited magnetization $\mathrm{M}_{\mathrm{xy}}(\mathbf{r}, \mathbf{v}, f)$ can be written as the Fourier transform of the RF $B_{1}$ field deposited on $k_{r^{-}} k_{V}-k_{f}$ space, where $k_{r}, k_{v}$, and $k_{f}$ are reciprocal Fourier variables of spatial position $\mathbf{r}$, velocity $\mathbf{v}$, and off-resonance $f$, respectively (8-10). 


$$
\begin{gathered}
M_{x y}(\mathbf{r}, \mathbf{v}, f)=i \gamma M_{0} \int_{0}^{T} B_{1}(t) e^{i 2 \pi\left[\mathbf{k}_{\mathbf{r}} \cdot \mathbf{r}+\mathbf{k}_{\mathbf{v}} \cdot \mathbf{v}+k_{f} f\right]} d t \\
\mathbf{k}_{\mathbf{r}}(t)=\frac{\gamma}{2 \pi} \int_{t}^{T} \mathbf{G}(s) d s, \mathbf{k}_{\mathbf{v}}(t)=\frac{\gamma}{2 \pi} \int_{t}^{T}(t-s) \mathbf{G}(s) d s, k_{f}(t)=T-t
\end{gathered}
$$

where $\gamma, M_{0}$ and $T$ are the gyromagnetic ratio, magnetization at equilibrium and pulse duration, respectively.

For velocity-selective but spatially non-selective excitation, hard RF sub-pulses are played out between a series of bipolar gradients each of which changes $\mathbf{k}_{\mathbf{v}}$ by

$\Delta \mathbf{k}_{\mathbf{v}}=\gamma / 2 \pi \int_{0}^{T_{b p}}\left(T_{b p}-t\right) \mathbf{G}_{\mathrm{bp}}(t) d t$ and $K_{f}$ by $\Delta k_{f}=T_{b p}$ (where $T_{b p}$ is the time duration of a bipolar gradient $\mathbf{G}_{\mathrm{bp}}(\mathrm{t})$ ), but does not change $\mathbf{k}_{\mathbf{r}}$. The envelope of RF sub-pulses determines the shape of an excitation profile in the $\mathbf{v}-f$ plane, and can be designed by the SLR transform (11). Aliased excitation occurs at increments of $\Delta \mathbf{k}_{\mathrm{v}}^{-1}$ (termed the velocity FOV) away from the desired excitation along the velocity axis since the $B_{1}$ field is deposited in the k-space in a discrete fashion. An excitation profile can be shifted along the velocity axis by applying an $\mathrm{RF}$ phase that is proportional to the first moment of a gradient waveform and the amount of velocity shifting $\mathbf{v}_{0}$.

$$
B_{1}(t) e^{j \alpha(t)}, \alpha(t)=\gamma \int_{t}^{T}(t-s) \mathbf{v}_{\mathbf{0}} \cdot \mathbf{G}(s) d s
$$

Figure 1 shows a VS saturation pulse sequence designed based on the k-space formalism, hereafter termed the reference VS sequence (a), and a Bloch simulation of the resultant longitudinal magnetization $M_{\mathrm{z}}$ over the $v$-f plane (b). In the plot of $M_{\mathrm{z}}$, a positive velocity indicates flow moving inferiorly (i.e., the direction of arterial flow in the legs). The design parameters were velocity FOV $=64 \mathrm{~cm} / \mathrm{s}$, full-width-half-maximum excitation bandwidth $=$ $17 \mathrm{~cm} / \mathrm{s}$, velocity shifting $v_{0}=-2 \mathrm{~cm} / \mathrm{s}$, and minimum phase filter for the SLR transform. On resonance, the VS pulse has a velocity pass-band of $[+5,+55] \mathrm{cm} / \mathrm{s}$ (defined as the velocity region for $M_{\mathrm{z}}>0.5 M_{0}$ ) and a velocity saturation-band of $[-4,0] \mathrm{cm} / \mathrm{s}$ (for $M_{\mathrm{z}}<$ $0.1 M_{0}$ ). These velocity pass- and saturation-bands would enable fairly good separation between arterial blood and background tissues (venous blood, muscle, synovial fluid) at a peak systolic phase in the calf. In the presence of off-resonance, however, the $M_{\mathrm{z}}$ profile is shifted along the velocity axis, which potentially degrades artery-to-background contrast. The profile shifting occurs at a rate of $0.15 \mathrm{~cm} / \mathrm{s} / \mathrm{Hz}$, as determined by the ratio between $k_{\mathrm{f}}$ and $k_{\mathrm{v}}$ increments by a bipolar gradient (i.e., $\Delta k_{t} / \Delta k_{V}=T_{b p} / \Delta k_{V}$ ) (10).

\section{Incorporating refocusing pulses}

To remove the off-resonance effect, we incorporate $180^{\circ}$ refocusing pulses into the reference VS pulse sequence and utilize phase refocusing as in spin-echo sequences. The bipolar gradient is split into two unipolar gradients between which a $180^{\circ}$ refocusing pulse is applied. The phase accrued by off-resonance during the period of the first unipolar will be cancelled out by the phase accrued in the opposite direction during the period of the second unipolar.

The incorporation of refocusing pulses necessitates some modifications of RF and gradient waveforms to achieve the desired velocity selectivity. First, a bipolar gradient should be designed with consideration of a time gap (of the duration of the $180^{\circ}$ pulse) between the halves of the bipolar. The same $\Delta \mathbf{k}_{\mathbf{v}}$ will be obtained by a pair of smaller unipolar gradients compared to the one that would have been needed without a time gap since $\mathbf{k}_{\mathbf{v}}$ keeps 
increasing during the time gap. Therefore, the increase in the overall pulse duration due to the inclusion of $180^{\circ}$ pulses will not be significantly large.

Second, re-sized unipolar gradients and an RF sub-pulse that are preceded by an oddnumbered $180^{\circ}$ pulse should be modified in a way that yields a $180^{\circ}$ rotated version of magnetization that would have been acquired without refocusing pulses. Then the subsequent even-numbered $180^{\circ}$ pulse will return the magnetization back to the same position as in the reference sequence. The required modifications are summarized as follow (see Appendix for derivations).

$$
\begin{gathered}
G_{u n i}(t) \rightarrow-G_{u n i}(t) \\
R F_{\text {sub }}(\alpha, \psi) \rightarrow R F_{\text {sub }}(\alpha, \pi-\psi)
\end{gathered}
$$

Where $G_{u n t}(t)$ and $R F_{\text {sub }}(\alpha, \psi)$ represent a unipolar gradient and an RF sub-pulse with a flip angle $\alpha$ and phase $\psi$ which would have been used without refocusing pulses. That is, the unipolar gradient should be inverted, and the RF sub-pulse should have the same flip angle $a$ but a modified phase of $\pi-\psi$. It should be noted that these changes are made only between an odd-numbered $180^{\circ}$ pulse and a subsequent even-numbered $180^{\circ}$ pulse.

Figure 2 shows a VS saturation pulse sequence designed by incorporating refocusing pulses, and a Bloch simulation of the resultant longitudinal magnetization. The same velocity FOV $(64 \mathrm{~cm} / \mathrm{s})$ and full-width-half-maximum excitation bandwidth $(17 \mathrm{~cm} / \mathrm{s})$ were used as in the reference design. A 1.2-ms-long composite pulse train of $90_{\mathrm{x}}-180_{\mathrm{y}}-90_{\mathrm{x}}$ was used for $B_{1^{-}}$ robust $180^{\circ}$ rotation (12). The unipolar gradients and RF sub-pulses between the $1^{\text {st }}$ and the $2^{\text {nd }}$ refocusing pulses and between the $3^{\text {rd }}$ and the $4^{\text {th }}$ refocusing pulses, are changed based on the modification rule (Eq. 3 ) as denoted by grey circles. The simulation results show that the effect of off-resonance on the $M_{\mathrm{z}}$ profile is significantly reduced compared to the reference VS sequence. Distortion of the $M_{\mathrm{z}}$ profile is noticeable in the presence of large off-resonance $(|f|>60 \mathrm{~Hz})$ which degrades the accuracy of the $180^{\circ}$ composite pulse.

\section{Tailoring VS sequence}

Unlike the reference VS sequence, an excitation profile yielded by the VS sequence with refocusing is not periodic over velocity whenever off-resonance is not zero. The aperiodicity is due to the fact that the spin's phase generated by a unipolar gradient and accordingly, the error caused by a subsequent inaccurate $180^{\circ}$ rotation, are different among spins whose velocities differ by multiples of the velocity FOV. We can leverage the aperiodicity for improving off-resonance robustness in a limited velocity ROI by perturbing sequence parameters that alter excitation errors in a velocity-dependent fashion.

Two design parameters are chosen to be numerically perturbed for tailoring the sequence. First, a time delay $T_{d}$ is added between a unipolar gradient and a $180^{\circ}$ pulse (represented by grey regions in Fig. 3a). The size of a unipolar gradient will decrease as $T_{d}$ increases, altering the sensitivity of excitation errors to velocity. Second, the excitation profile is shifted along the velocity axis by multiples of the velocity FOV using the RF phase modulation (Eq. 2). That is, the fidelity of the excitation profile is examined near the mainlobe as well as sidelobes. The iterative search for a local optimal solution can be represented as 


$$
\begin{aligned}
& \underset{\Delta v, T_{d}}{\operatorname{argmin}} \max _{v \in v_{R O I}}\left|M_{z}\left(\Delta v, T_{d} ; v, f\right)-M_{z}^{r e f}(v)\right| \\
& f \in f_{\text {ROI }}
\end{aligned}
$$

Where $M_{\mathrm{z}}$ is a longitudinal magnetization profile determined by the time delay $T_{d}$ and the amount of velocity shifting $\Delta v$, and $M_{\mathrm{Z}}{ }^{r e f}$ is the reference profile obtained with the assumption of on-resonance $(f=0 \mathrm{~Hz})$. The sequence parameters $T_{d}$ and $\Delta v$ were discretized to $[0,8,16, \ldots, 296,304] \times 4 \mu$ s and $\left[0, \pm \mathrm{FOV}_{\mathrm{v}}, \pm 2 \mathrm{FOV}_{\mathrm{v}}, \pm 3 \mathrm{FOV}_{\mathrm{v}}\right] \mathrm{cm} / \mathrm{s}$, respectively. The spin parameters $V$ and $f$ were discretized to $[-3,-2, \ldots, 49,50] \mathrm{cm} / \mathrm{s}$ and $[-80,-70, \ldots, 70$, $80 \mathrm{~Hz}$, respectively. Note that $v$ and $f$ varied within limited ranges that are expected in the lower extremities.

Figure 3 contains a VS saturation pulse sequence tailored using the parameters $T_{d}=416 \mu \mathrm{s}$ and $\Delta v=-\mathrm{FOV}_{\mathrm{V}}$ that were found through the iterative search. This VS sequence is our final design used for all in vivo MRA experiments performed in this study. The simulated $M_{\mathrm{Z}}$ profiles demonstrate that off-resonance immunity is further improved in the velocity ROI $([-3,+50] \mathrm{cm} / \mathrm{s})$ compared to the design without tailoring particularly in the presence of large off-resonance (Figs. 2b-d vs Figs. 3b-d). Figure 4 shows simulated $M_{\mathrm{z}}$ profiles across varying $B_{1}$ scale factors with three off-resonance values $(f=-60,0,60 \mathrm{~Hz})$. As the $B_{1}$ scale increases, the longitudinal magnetization in the velocity stop-band of interest decreases ([ -3 , $0] \mathrm{cm} / \mathrm{s}$ ) due to increasing actual $B_{1}$ values of the RF sub-pulses. The same behavior is expected in $M_{\mathrm{z}}$ profiles from the reference VS sequence. As the $B_{1}$ scale deviates from one, undesired excitation occurs in the velocity pass-band of interest $([+5,+50] \mathrm{cm} / \mathrm{s})$ due to inaccurate $180^{\circ}$ refocusing rotations, but only marginally in a $B_{1}$ scale range of 0.85 to 1.15 .

\section{In vivo experiments}

In vivo experiments were performed in seven healthy subjects and a patient for the purpose of comparing off-resonance sensitivity of the reference and proposed VS saturations (with refocusing and tailoring) and demonstrating the feasibility of using the proposed VS saturation preparation for NCE peripheral MRA. All subjects provided written consent forms approved by our institutional review board. A $1.5 \mathrm{~T}$ whole-body MR system was used (Signa HDx; GE Healthcare, Waukesha, WI). The MRA pulse sequence was cardiac triggered by peripheral or ECG gating, and consisted of a VS saturation pulse, a spectrally selective fat saturation pulse, and a 3DFT balanced SSFP (bSSFP) readout (Fig. 5). The VS saturation pulse was played at the time of peak systolic flow by adjusting a cardiac trigger delay (TD) based on prior phase-contrast (PC) flow measurements. The 3DFT acquisition used centric and linear view orders along phase- and partition-encoding directions, respectively.

Per subject, 2D PC flow MRI was performed with an axial scan plane. Through-plane flow was measured in the anterior tibial artery, femoral artery, or iliac artery depending on the S/I level of the imaging volume, and displayed over cardiac phases to find a trigger delay for the maximum systolic flow. Imaging parameters were $\mathrm{VENC}=80 \mathrm{~cm} / \mathrm{s}$, spatial resolution $=$ $1.1 \times 1.1 \mathrm{~mm}^{2}, \mathrm{FOV}=14 \times 14 \mathrm{~cm}^{2}$, slice thickness $=6 \mathrm{~mm}$, view per segment $=2$, and temporal resolution $=20.6 \mathrm{~ms}$.

To evaluate off-resonance sensitivity under the control of various $B_{0}$ offsets, the right leg was imaged with a small imaging FOV in a subject. A lower extremity coil designed for a single leg was used for signal reception. The reference and proposed VS saturation pulses with the same pass-band of $[+5,+55] \mathrm{cm} / \mathrm{s}$ and saturation-band of $[-4,0] \mathrm{cm} / \mathrm{s}$ were used for comparison (Figs. 1 and 3, respectively). Imaging parameters were imaging orientation = axial, flip angle $\alpha=70^{\circ}, \mathrm{FOV}=15 \times 15 \times 3.9 \mathrm{~cm}^{3}$ (in the order of $\mathrm{L} / \mathrm{R}, \mathrm{A} / \mathrm{P}$, and $\mathrm{S} / \mathrm{I}$ 
directions), spatial resolution $=1.1 \times 1.1 \times 1.3 \mathrm{~mm}^{3}$, number of axial slices $=30, \mathrm{TR}=4.6 \mathrm{~ms}$, readout bandwidth $=125 \mathrm{kHz}$, raised cosine-windowed ramp for 8 catalyzation excitations, number of phase encodes per acquisition block $=68$, acquisition time per acquisition block $=313 \mathrm{~ms}$, and scan time $=60$ heart beats $(51 \mathrm{sec}$ based on 70 beats $/ \mathrm{min})$. The first scan was performed under a well-shimmed condition determined by the standard GE automatic shimming protocol. Four additional scans were performed after the center frequency was manually changed by $-80,-40,40$, and $80 \mathrm{~Hz}$.

The reference and proposed VS preparations were also compared within a MRA protocol for imaging the calves with a large FOV in four healthy subjects. An eight-channel cardiac coil array was used for signal reception. Imaging parameters were imaging orientation $=$ coronal, flip angle $\alpha=70^{\circ}$, spatial resolution $=1.1 \times 1.1 \times 1.3 \mathrm{~mm}^{3}, \mathrm{FOV}=30 \times 32 \times 9.1 \mathrm{~cm}^{3}$ (in the order of S/I, L/R, and A/P directions), number of coronal slices $=70, \mathrm{TR}=4.5 \mathrm{~ms}$, readout bandwidth $=125 \mathrm{kHz}$, number of phase encodes per acquisition block $=73$, acquisition time per acquisition block $=329 \mathrm{~ms}$, and scan time $=256$ heart beats $(3.7 \mathrm{~min}$ based on 70 beats $/$ min). A field map was acquired using two GRE acquisitions with an echo delay of $1.5 \mathrm{~ms}$ and a two-fold lower spatial resolution $\left(2.2 \times 2.2 \times 2.6 \mathrm{~mm}^{3}\right)$. Artery-to-vein contrast-to-noiseratio (CNR) and artery-to-muscle CNR were measured in raw 3D MRA images. Per leg, arterial signal intensities were obtained from 7 ROIs manually drawn on the popliteal artery (1 ROI), anterior tibial artery (2 ROIs), posterior tibial artery (2 ROIs), and peroneal artery (2 ROIs). Venous signal was estimated in a similar way from 7 ROIs drawn on the deep vein near the arterial ROIs. Image noise was estimated by the standard deviation of the air signal in 2 ROIs placed outside the legs.

To demonstrate large angiographic coverage of the proposed MRA method, the pelvis, thighs and calves were scanned using three stations in two healthy subjects. The imaging parameters for the three stations were spatial resolution $=1.3 \times 1.3 \times 2.0 \mathrm{~mm}^{3} / 1.3 \times 1.3 \times 1.8$ $\mathrm{mm}^{3} / 1.1 \times 1.1 \times 1.3 \mathrm{~mm}^{3}$ (pelvis/thighs/calves), $\mathrm{FOV}=30 \times 34 \times 16 \mathrm{~cm}^{3} / 30 \times 34 \times 13.7$ $\mathrm{cm}^{3} / 30 \times 32 \times 8.3 \mathrm{~cm}^{3}$, number of phase encodes per acquisition block $=65 / 65 / 73$, and scan time $=320$ heart beats $/ 304$ heart beats $/ 256$ heart beats $(4.5 \mathrm{~min} / 4.3 \mathrm{~min} / 3.7 \mathrm{~min}$ based on 70 beats/min). The upper bound of the velocity pass-band initially designed for the calves (55 $\mathrm{cm} / \mathrm{s}$ ) was increased by $15 \%$ and $30 \%$ for the thighs and pelvis, respectively, to accommodate higher arterial flow in the two superior stations. This adjustment was done simply by scaling the initial VS gradient waveform (Fig. 6). The proposed MRA was also performed in a patient who was referred for a digital subtraction angiography (DSA) examination. The patient's thighs and calves were scanned using two stations each of which had the same imaging parameters described above.

\section{Results}

Figure 7 shows results of testing the off-resonance sensitivity of the reference (a) and proposed (b) VS saturation sequences. A reference image acquired without VS preparation (c) and a field map obtained under the well-shimmed condition (d) are also shown. Both reference and proposed VS saturations yield excellent artery-to-vein and artery-to-muscle contrast under the well-shimmed condition (the $3^{\text {rd }}$ columns in a and $b$ ). With increasing absolute value of the center frequency offset $(\triangle \mathrm{CF})$, however, background signal in the images from the reference VS saturation increases due to the excitation profile shifting. In the images from $\triangle \mathrm{CF}= \pm 80 \mathrm{~Hz}$, image artifacts are also observed (denoted by open arrows) since the transient oscillations in bSSFP imaging are large near the null-band $( \pm 108.7 \mathrm{~Hz}$ with $\mathrm{TR}=4.6 \mathrm{~ms}$ ) when the initial magnetization is substantially different from the steadystate magnetization $\left(M_{\mathrm{z}}=0.02 M_{0}\right.$ with $f= \pm 80 \mathrm{~Hz}, T_{1}=870 \mathrm{~ms}, T_{2}=50 \mathrm{~ms}$, and TR $=4.6$ $\mathrm{ms})$. Background signal in the images from the proposed VS saturation remains nearly the same for all tested $\triangle \mathrm{CF}$ s due to the off-resonance immunity. The transient bSSFP artifact is 
negligible in the presence of large off-resonance as the initial magnetization of background tissues is small and similar to the steady-state magnetization.

Figure 8 contains representative angiograms of the calves using the reference and proposed VS preparations and the field maps of the center coronal slices in two healthy subjects. Reformatted coronal maximum-intensity-projection (MIP) and raw axial images from the reference VS preparation have high background intensities in the regions of large offresonance due to the VS excitation profile shifting (a,c,d,f,h). The MIP and raw images from the proposed VS preparation show much more uniform background suppression across the entire FOV $(b, e, g)$. This was achieved over a wide range of off-resonance as seen by the field maps, which demonstrates the off-resonance robustness of the proposed VS saturation. A slight increase in the background signal is observed in the regions with large superior/ inferior offsets, which is most likely due to $\mathrm{B}_{1}+$ variation (open arrows).

The field map and CNR measurements are summarized in Table 1. Off-resonance was measured as $-3.2 \pm 38.2 \mathrm{~Hz}$ across four subjects, which resulted in a $95 \%$ confidence interval of $[-78.1,+71.7] \mathrm{Hz}$. Artery-to-vein CNR was $61.7 \pm 22.7$ (mean \pm standard deviation) and $71.0 \pm 11.4$ with the reference and proposed VS preparations, respectively, across the four subjects. Artery-to-muscle CNR was $58.5 \pm 27.8$ and $75.3 \pm 12.1$ with the reference and proposed VS preparation, respectively. The angiograms obtained using the proposed preparation yielded a larger mean value and a smaller standard deviation in each of the two CNRs due to lower and more uniform signal intensities of the vein and muscle over the FOV.

Figure 9 shows peripheral angiograms using three stations for the pelvis, thighs and calves in two healthy subjects. Iliac, femoral, popliteal, and tibial arteries are clearly delineated across a total of $90 \mathrm{~cm}-\mathrm{S} / \mathrm{I} \mathrm{FOV}$ using the scaled VS gradients for each of the three stations (Fig. 6). The angiograms also show excellent suppression of the vein, muscle, and synovial fluid in all three stations. The residual fat signal in the calf station of the first subject is due to large field inhomogeneity which shifts the fat frequency beyond the spectral coverage of the fat saturation pulse.

Figure 10 shows the result of an initial patient study with a comparison to DSA. The mild narrowing of the right femoral artery and occlusion of the right popliteal artery are well identified on both the MR and DSA images (arrows). While all major arteries are identified on the MR images, most small vessel branches appear to be less clear than the DSA images (black arrowheads) presumably due to lower spatial resolution and slow velocity of arterial blood. Note that the right anterior tibial artery is better visualized by NCE MRA as the concentration of X-ray contrast for DSA is expected to be limited in arteries distal to the occlusion (white arrowhead) (13).

\section{Discussion}

Velocity-selective excitation is a promising magnetization preparation for NCE MRA. By saturating all magnetizations except arterial blood moving in a particular direction and speed, high artery-to-background contrast can be achieved using a single acquisition without subtraction. Because VS magnetization preparation is spatially non-selective, 3D encoding can be used with a large FOV and high spatial resolution in all three dimensions.

Dependence only on systolic flow is also advantageous over existing flow-sensitive MRA methods that require data acquisition at both systole and diastole (14-17) since the systolic interval is less affected by $R-R$ variability or arrhythmia $(18,19)$.

A VS excitation pulse sequence for given velocity pass- and stop-bands can be designed using the generalized excitation k-space formalism in a straightforward manner. However, 
the resultant excitation profile is shifted along velocity in proportion to off-resonance. Therefore, we proposed a new VS excitation sequence which significantly reduces the offresonance-induced excitation error in a velocity ROI by incorporating refocusing pulses and tailoring sequence parameters. According to the numerical simulations, the proposed design achieves off-resonance immunity of approximately $\pm 80 \mathrm{~Hz}$. Accordingly, in vivo peripheral MRA using the proposed VS saturation preparation yielded high artery-to-background contrast over a wide range of field inhomogeneity.

The proposed VS saturation-prepared NCE MRA involves some sequence parameters that remain to be further optimized. The velocity pass-band might need to be widened to better include very slow or turbulent flow potentially in patients with artery disease. The lower bound of the pass-band can be reduced by simply scaling up the VS gradient waveforms but at the expense of a decrease in the upper bound. An increase in the VS pulse duration is inevitable to reduce the lower bound while maintaining the same upper bound or increasing it. Addition of a time delay between the VS preparation and data acquisition may be helpful for visualizing slow flow due to the time-of-flight effect. In this case, the VS excitation flip angle should be increased appropriately beyond $90^{\circ}$ to compensate for $T_{1}$ recovery of background tissues. The number of $180^{\circ}$ pulses (or RF sub-pulses) should be chosen as a trade-off between the sharpness of the velocity transition-band and excitation accuracy which is affected by inaccurate $180^{\circ}$ rotations and high-order motion of blood. A composite $180^{\circ}$ pulse itself has potential variants. While a train of $90_{x}-180_{y}-90_{x}$ appeared to be sufficiently robust against $B_{0}$ and $B_{1}$ variations in our peripheral MRA studies, other combinations can be chosen to flexibly trade off $B_{0}$ sensitivity, $B_{1}$ sensitivity and the pulse duration $(20,21)$.

The PC flow imaging and subsequent time curve analysis for finding the optimal trigger delay (TD) for VS preparation took approximately $1.5 \mathrm{~min}$. To reduce the scan time, one can use rapid spiral-based flow imaging which is expected to be less than 5 heart-beats long based on previous studies $(22,23)$. To reduce the analysis time and avoid manual intervention, a small ROI can be swept through the 3D $x-y-t$ space to automatically find its spatial and temporal indices that yield the maximum velocity value.

Ideally, it would be desirable to play the VS preparation at the peak systolic phase to ensure large arterial flow. However, the timing of peak systolic flow varies along the length of the artery and also between two legs particularly in cases of arterial pathology. This issue is associated with a range of (sub-optimal) TDs at which arterial flow velocity is still larger than the lower bound of the velocity pass-band $(5 \mathrm{~cm} / \mathrm{s}$ in the calf station). TDs outside such a tolerable range would cause signal loss in arteries. In this study, we determined the VS preparation timing based on flow measurement at iso-center (i.e., in the middle of the imaging volume) to balance potential deviations from the optimal timings across the FOV. While the trigger delay determined at iso-center yielded fairly uniform visualization of arteries in all tested subjects including a patient with unilateral disease (Fig. 10), further investigation is needed to estimate the tolerance and effect of sub-optimal TDs in a large cohort of subjects.

Alternative NCE MRA methods have recently been developed that utilize MR sensitivity to arterial flow. Quiescent-interval single shot (QISS) imaging is an inflow-based 2D multislice approach, which employs slice-selective pre-saturation for background suppression and time-of-flight effect during the subsequent delay (quiescent interval) for artery visualization (24). QISS is robust to a wide range of arterial flow patterns due to short S/I paths required for fresh arterial blood to traverse during the saturation delay, and robust to motion due to the use of 2D multi-slice imaging. As a trade-off, it would be challenging to use large-FOV $3 \mathrm{D}$ encoding which is beneficial for high SNR and high spatial resolution along the S/I 
direction. Another flow-sensitive NCE MRA method used a pulse sequence of $90_{\mathrm{x}^{-}} G-180_{\mathrm{y}^{-}}$ $G-90_{-x}$ (i.e., a refocused version of the $90_{\mathrm{x}^{-}} G-90_{-\mathrm{x}}$ module) as magnetization preparation $(16,17)$. The magnetization response over velocity is sinusoidal for each isochromat, but with the assumption of laminar flow and consideration of intravoxel dephasing, the response becomes approximately a sinc function of the slope of velocity variation and the first moment of gradient $(16,25)$. The resultant black-artery image can be subtracted from a reference image to generate a bright-artery angiogram. The subtractive nature of the method enables perfect background suppression and therefore superior artery-to-background contrast (22), but involves issues of increased scan time and motion effects.

\title{
Conclusions
}

We designed and tested a new off-resonance-robust VS magnetization preparation pulse for non-subtractive NCE peripheral MRA. The excitation error due to off-resonance can be reduced by incorporating $180^{\circ}$ refocusing pulses into a reference k-space-based design, and further suppressed in a velocity ROI by tailoring sequence parameters. Consistent with the numerical simulations, NCE MRA using the proposed VS preparation was able to create high angiographic contrast more reliably than the reference VS preparation in the presence of field inhomogeneity ranging from -78 to $72 \mathrm{~Hz}$ in the calves. While clinical feasibility was shown by successful identification of arterial stenosis in a patient, the performance of the proposed technique in cases of arterial pathology needs to be further investigated.

\section{Acknowledgments}

\author{
Grant Sponsors: \\ National Institutes of Health (5R01 HL075803) \\ GE Healthcare
}

\section{References}

1. Nishimura DG, Macovski A, Pauly JM. Magnetic resonance angiography. IEEE Trans Med Imaging. 1986; MI-5(3):140-151. [PubMed: 18244000]

2. Pauly, J.; Nishimura, DG.; Macovski, A. Cancellation excitation for angiography. Proceedings of the 5th SMRM Annual Meeting; 1986; Montreal, Canada. p. 70-71.

3. Moran PR, Saloner D, Tsui BMW. NMR velocity-selective excitation composites for flow and motion imaging and suppression of static tissue signal. IEEE Trans Med Imaging. 1987; MI-6(2): 141-147. [PubMed: 18230440]

4. Lee JN, Parker DL. MR angiography with adiabatic flow excitation. J Magn Reson Imaging. 1992; 2(4):431-436. [PubMed: 1633396]

5. Korosec FR, Grist TM, Polzin JA, Weber DM, Mistretta CA. MR angiography using velocityselective preparation pulses and segmented gradient-echo acquisition. Magn Reson Med. 1993; 30(6):704-714. [PubMed: 8139452]

6. Pope JM, Yao S. Flow-selective pulse sequences. Magn Reson Imaging. 1993; 11(4):585-591. [PubMed: 8316071]

7. Norris DG, Schwarzbauer C. Velocity selective radiofrequency pulse trains. J Magn Reson. 1999; 137(1):231-236. [PubMed: 10053152]

8. de Rochefort L, Maître X, Bittoun J, Durand E. Velocity-selective RF pulses in MRI. Magn Reson Med. 2006; 55(1):171-176. [PubMed: 16342055]

9. Pauly J, Nishimura DG, Macovski A. A k-space analysis of small-tip-angle excitation. J Magn Reson. 1989; 81:43-56. 
10. Shin T, Worters PW, Hu BS, Nishimura DG. Non-contrast-enhanced renal and abdominal MR angiography using velocity-selective inversion preparation. Magn Reson Med. 10.1002/mrm. 24356.

11. Pauly J, Le Roux P, Nishimura DG, Macovski A. Parameter relations for the Shinnar-Le Roux selective excitation pulse design algorithm. IEEE Trans Med Imaging. 1991; 10(1):53-65. [PubMed: 18222800]

12. Levitt MH, Freeman R. NMR population inversion using a composite pulse. J Magn Reson. 1979; 33:473-476.

13. Baum RA, Rutter CM, Sunshine JH, Blebea JS, Blebea J, Carpenter JP, Dickey KW, Quinn SF, Gomes AS, Grist TM, McNeil BJ. Multicenter trial to evaluate vascular magnetic resonance angiography of the lower extremity. JAMA. 1995; 274:875-880. [PubMed: 7674500]

14. Meuli RA, Wedeen VJ, Geller SC, Edelman RR, Frank LR, Brady TJ, Rosen BR. MR gated subtraction angiography: evaluation of lower extremities. Radiology. 1986; 159(2):411-418. [PubMed: 3961174]

15. Miyazaki M, Sugiura S, Tateishi F, Wada H, Kassai Y, Abe H. Non-contrast-enhanced MR angiography using 3D ECG-synchronized half-Fourier fast spin echo. J Magn Reson Imaging. 2000; 12(5):776-783. [PubMed: 11050650]

16. Fan Z, Sheehan J, Bi X, Liu X, Carr J, Li D. 3D noncontrast MR angiography of the distal lower extremities using flow-sensitive dephasing (FSD)-prepared balanced SSFP. Magn Reson Med. 2009; 62(6):1523-1532. [PubMed: 19877278]

17. Priest AN, Graves MJ, Lomas DJ. Non-contrast-enhanced vascular magnetic resonance imaging using flow-dependent preparation with subtraction. Magn Reson Med. 2012; 67(3):628-637. [PubMed: 21713977]

18. Weissler AM, Harris WS, Schoenfeld CD. Systolic time intervals in heart failure in man. Circ. 1968; 37(2):149-159.

19. Gharib AM, Herzka DA, Ustun AO, Desai MY, Locklin J, Pettigrew RI, Stuber M. Coronary MR angiography at 3T during diastole and systole. J Magn Reson Imaging. 2007; 26:921-926. [PubMed: 17896391]

20. Levitt MH. Composite pulses. Progr NMR Spectrosc. 1986; 18:61-122.

21. Levitt, MH. Composite pulses. In: Grant, DM.; Harris, RK., editors. Encyclopedia of nuclear magnetic resonance. New York: Willey; 1996.

22. Nayak KS, Pauly JM, Kerr AB, Hu BS, Nishimura DG. Real-time color flow MRI. Magn Reson Med. 2000; 43(2):251-258. [PubMed: 10680689]

23. Nayak KS, Hu BS. Triggered real-time MRI and cardiac applications. Magn Reson Med. 2003; 49(1):188-192. [PubMed: 12509837]

24. Edelman RR, Sheehan JJ, Dunkle E, Schindler N, Carr J, Koktzoglou I. Quiescent-interval singleshot unenhanced magnetic resonance angiography of peripheral vascular disease: Technical considerations and clinical feasibility. Magn Reson Med. 2010; 63(4):951-958. [PubMed: 20373396]

25. Haacke, EM.; Brown, RW.; Thompson, MR.; Venkatesan, R. Magnetic resonance imaging physical principles and sequence design. New York: Wiley-Liss; 1999.

\section{Appendix}

\section{Modification of gradient and RF pulses in refocused VS excitation}

We derive the modification of unipolar gradients and RF sub-pulses required by the inclusion of refocusing pulses in a VS excitation sequence. The specific requirement is that the magnetization in the refocused design should be a $180^{\circ}$ rotated version of the magnetization that would have been obtained in the original design without refocusing pulses during a period between an odd-numbered $180^{\circ} \mathrm{RF}$ pulse and a next coming evennumbered $180^{\circ}$ RF pulse. Below, $\mathbf{R}_{\mathrm{x}}(\cdot), \mathbf{R}_{\mathrm{y}}(\cdot)$ and $\mathbf{R}_{\mathrm{z}}(\cdot)$ denote rotation matrices around the $x, y$, and $z$ axis, respectively. 
An arbitrary magnetization immediately before a unipolar gradient in the original design is denoted as $M_{1}$. A magnetization immediately before a corresponding unknown unipolar gradient in the refocused design is assumed to be a $180^{\circ}$ rotated version of $\mathbf{M}_{1}$, i.e., $\mathbf{R}_{\mathrm{y}}(\pi) \mathbf{M}_{1}$. The magnetization immediately after the unknown unipolar gradient in the refocused design should still be a $180^{\circ}$ rotated version of the magnetization after the unipolar in the original design.

$$
\mathbf{R}_{z}(\beta) \mathbf{R}_{y}(\pi) \mathbf{M}_{1}=\mathbf{R}_{y}(\pi) \mathbf{R}_{z}(\theta) \mathbf{M}_{1} \quad[\mathrm{~A} 1]
$$

where $\theta$ and $\beta$ represent the phases generated by the unipolar gradient in the original design, and by the unknown unipolar in the refocused design, respectively.

Simplifying Eq. A1 gives

$$
\mathbf{R}_{z}(\beta)=\mathbf{R}_{y}(\pi) \mathbf{R}_{z}(\theta) \mathbf{R}_{y}(-\pi)=\mathbf{R}_{z}(-\theta) \quad[\mathrm{A} 2]
$$

That is, the unipolar gradient in the refocused design should be an inverted version of the original unipolar gradient.

Similar to the derivation for unipolar gradients, magnetizations immediately before RF subpulses in the original and refocused designs are denoted as $\mathbf{M}_{2}$ and $\mathbf{R}_{\mathrm{y}}(\pi) \mathbf{M}_{2}$, respectively. Excitation by an RF sub-pulse with a flip angle $\alpha$ and phase $\psi$ in the original design can be represented by $\mathbf{R}_{\mathrm{Z}}(-\psi) \mathbf{R}_{\mathrm{x}}(\alpha) \mathbf{R}_{\mathrm{Z}}(\psi)$. Since the magnetization immediately after the unknown RF sub-pulse in the refocused design should be a $180^{\circ}$ rotated version of the magnetization after the RF sub-pulse in the original design,

$$
\mathbf{B R}_{y}(\pi) \mathbf{M}_{2}=\mathbf{R}_{y}(\pi) \mathbf{R}_{z}(-\psi) \mathbf{R}_{x}(\alpha) \mathbf{R}_{z}(\psi) \mathbf{M}_{2} \quad[\mathrm{~A} 3]
$$

where $\mathbf{B}$ denotes the unknown rotation by the RF sub-pulse in the refocused design, and is written as

$$
\mathbf{B}=\mathbf{R}_{y}(\pi) \mathbf{R}_{z}(-\psi) \mathbf{R}_{x}(\alpha) \mathbf{R}_{z}(\psi) \mathbf{R}_{y}(-\pi) \quad[\mathrm{A} 4]
$$

Using $\mathbf{R}_{Z}(\psi) \mathbf{R}_{y}(-\pi)=\mathbf{R}_{X}(\pi) \mathbf{R}_{Z}(\pi-\psi)$ and $\mathbf{R}_{y}(\pi) \mathbf{R}_{Z}(-\psi)=\mathbf{R}_{Z}(-\pi+\psi) \mathbf{R}_{X}(-\pi)$, Eq. A4 becomes

$$
\begin{gathered}
\mathbf{B}=\mathbf{R}_{z}(-\pi+\psi) \mathbf{R}_{x}(-\pi) \mathbf{R}_{x}(\alpha) \mathbf{R}_{x}(\pi) \mathbf{R}_{z}(\pi-\psi) \\
=\mathbf{R}_{z}(-\pi+\psi) \mathbf{R}_{x}(\alpha) \mathbf{R}_{z}(\pi-\psi)
\end{gathered}
$$

That is, the RF sub-pulse in the refocused design should have the same flip angle $a$ and a modified phase of $\pi-\psi$. 

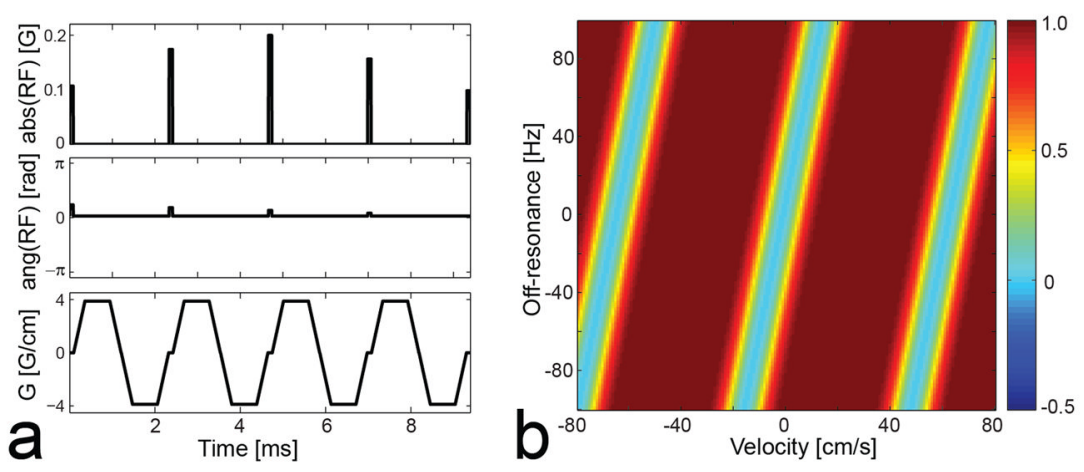

Figure 1.

Reference VS saturation pulse sequence designed based on the excitation k-space formalism (a) and simulated longitudinal magnetization $M_{\mathrm{z}}$ over the $v-f$ plane (b). In the figure of simulated $M_{\mathrm{Z}}$, a positive velocity indicates flow moving inferiorly (i.e., the direction of arterial flow in the legs). On resonance, the VS sequence saturates magnetization moving at 0 to $4 \mathrm{~cm} / \mathrm{s}$ in the superior direction, which includes stationary tissues and venous blood, but barely affects magnetization moving at 10 to $50 \mathrm{~cm} / \mathrm{s}$ in the inferior direction, which includes arterial blood at a peak systolic phase. However, the $M_{\mathrm{z}}$ profile is shifted along velocity in proportion to off-resonance $(0.15 \mathrm{~cm} / \mathrm{s} / \mathrm{Hz})$, which potentially degrades artery-tobackground contrast. 

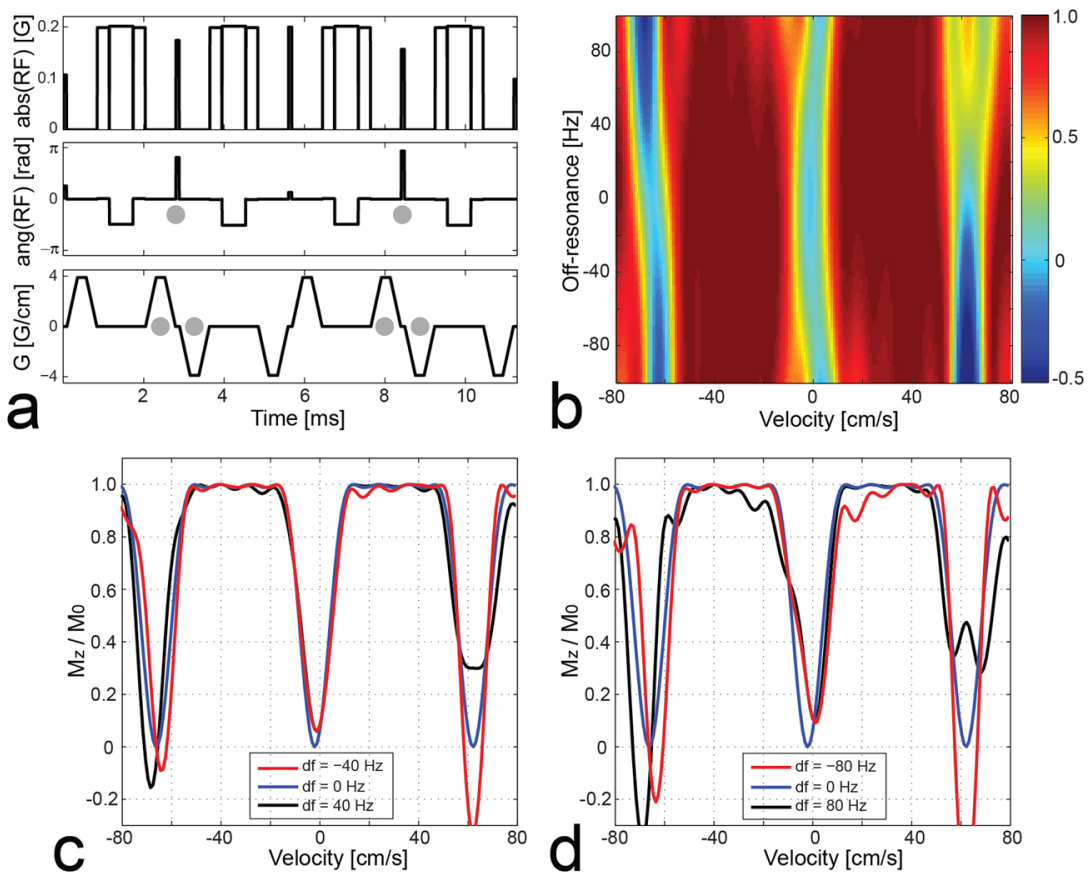

Figure 2.

VS saturation pulse sequence with refocusing (a), simulated longitudinal magnetization $M_{\mathrm{Z}}$ over the $v$ - $f$ plane (b) and for off-resonance $f=0, \pm 40, \pm 80 \mathrm{~Hz}$ (c, d). Each bipolar gradient is split into two unipolar gradients, and a $90_{\mathrm{x}}-180_{\mathrm{y}}-90_{\mathrm{x}}$ composite pulse is inserted between them. The phases of RF sub-pulses and unipolar gradients are modified according to Eq. 3 in the periods between the $1^{\text {st }}$ and $2^{\text {nd }}$ refocusing pulses and between the $3^{\text {rd }}$ and $4^{\text {th }}$ refocusing pulses (denoted by grey circles). The off-resonance sensitivity is significantly reduced compared to the reference design due to the phase refocusing by the $180^{\circ}$ pulses. The composite pulse itself is sensitive to off-resonance, which causes noticeable degradation of the $M_{\mathrm{z}}$ profile in the presence of large off-resonance $(|f|>60 \mathrm{~Hz})$. 

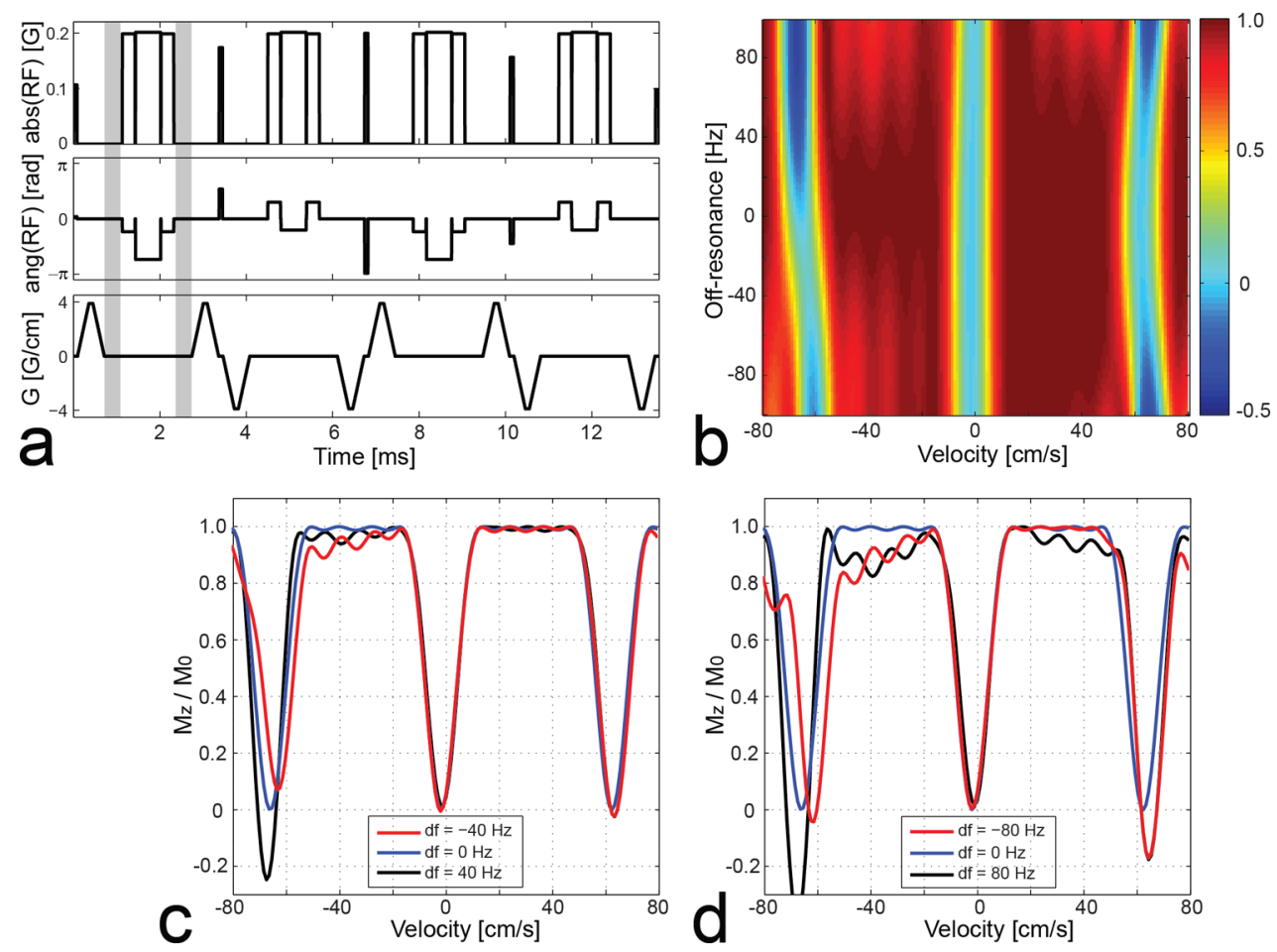

Figure 3.

VS saturation pulse sequence with refocusing and tailoring (a), simulated longitudinal magnetization $M_{\mathrm{z}}$ over the $v$ - $f$ plane (b) and for off-resonance $f=0, \pm 40, \pm 80 \mathrm{~Hz}$ (c, d). A time delay $T_{d}$ (denoted by grey bars) and profile shifting along the velocity axis $\Delta V$ were iteratively found that maximize the fidelity of an $M_{\mathrm{z}}$ profile in a velocity region $([-3,+50]$ $\mathrm{cm} / \mathrm{s})$ and an off-resonance range $([-80,+80] \mathrm{Hz})$ expected in the lower extremities. The resulting $M_{\mathrm{z}}$ profiles show that off-resonance immunity is further improved in the prescribed velocity region of interest compared to the VS sequence without tailoring particularly when off-resonance is large. 


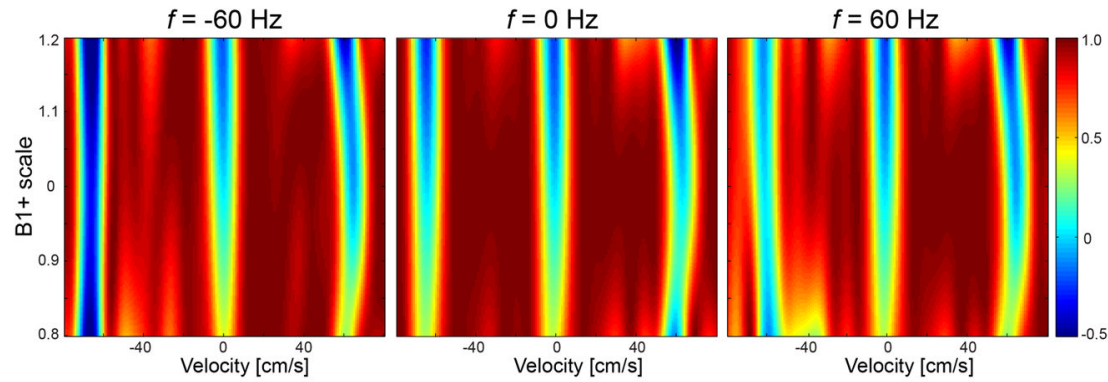

Figure 4.

Sensitivity of the proposed VS saturation sequence to $B_{1}+$ variation. Longitudinal magnetization in the velocity stop-band of interest $([-3,0] \mathrm{cm} / \mathrm{s})$ decreases with increasing $B_{1}$ scale due to increasing actual $B_{1}$ values of RF sub-pulses. Magnetization in the velocity pass-band of interest $([+5,+50] \mathrm{cm} / \mathrm{s})$ is undesirably excited due to inaccurate $180^{\circ}$ rotations by $B_{1}$ scaled composite pulses, but only marginally in a $B_{1}$ scale region of 0.85 to 1.15 . 

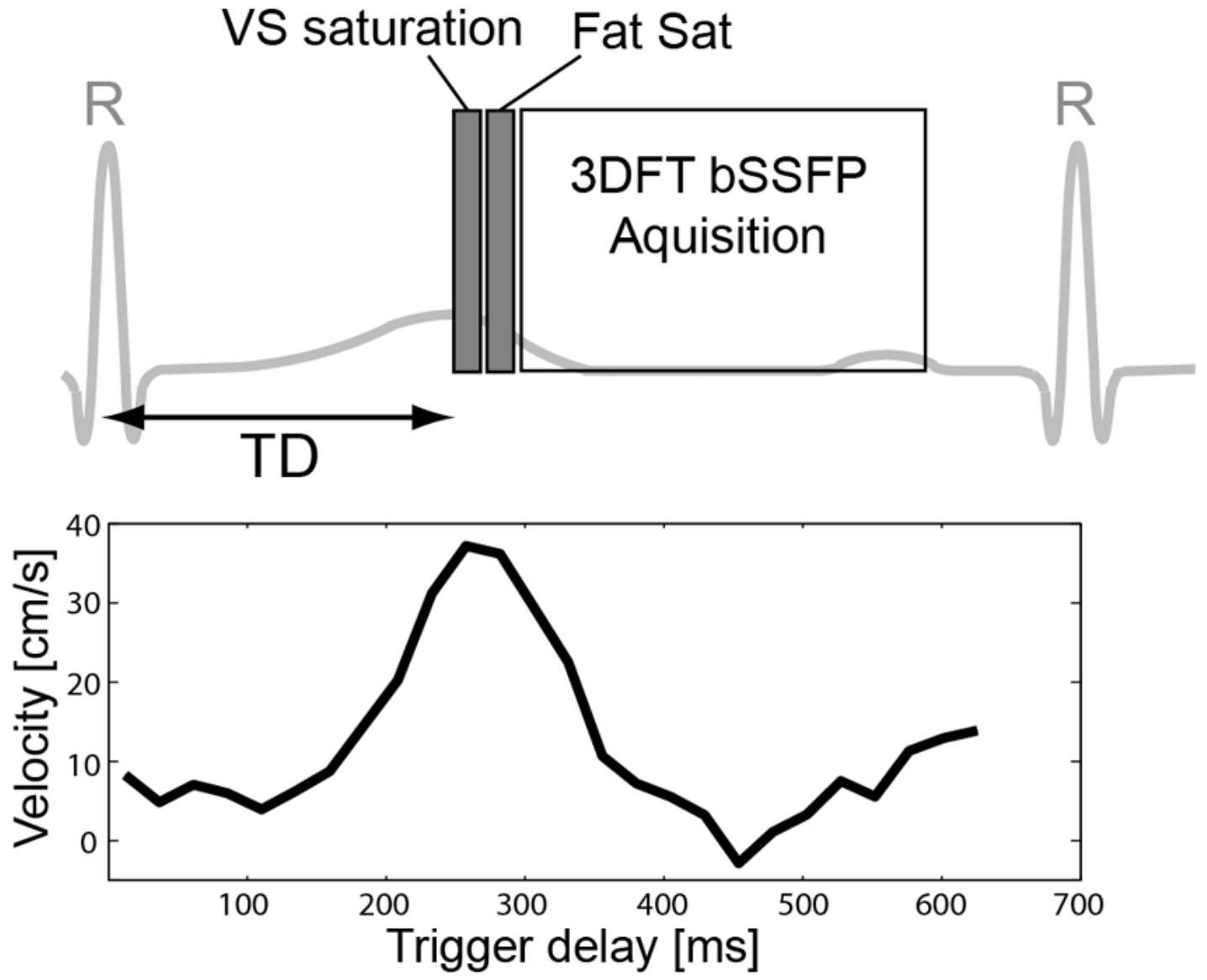

Figure 5.

Timing diagram of the VS saturation-prepared peripheral MRA sequence (top). The sequence was cardiac triggered by peripheral gating, and consisted of a VS saturation pulse, a spectrally selective fat saturation pulse, and a segmented balanced SSFP 3DFT readout with centric and linear orders along phase- and partition-encoding directions, respectively. The VS saturation pulse was played at the time of peak systolic flow by adjusting a trigger delay (TD) based on prior phase-contrast flow MRI. A representative velocity measurement in the anterior tibial artery is shown at the bottom. 

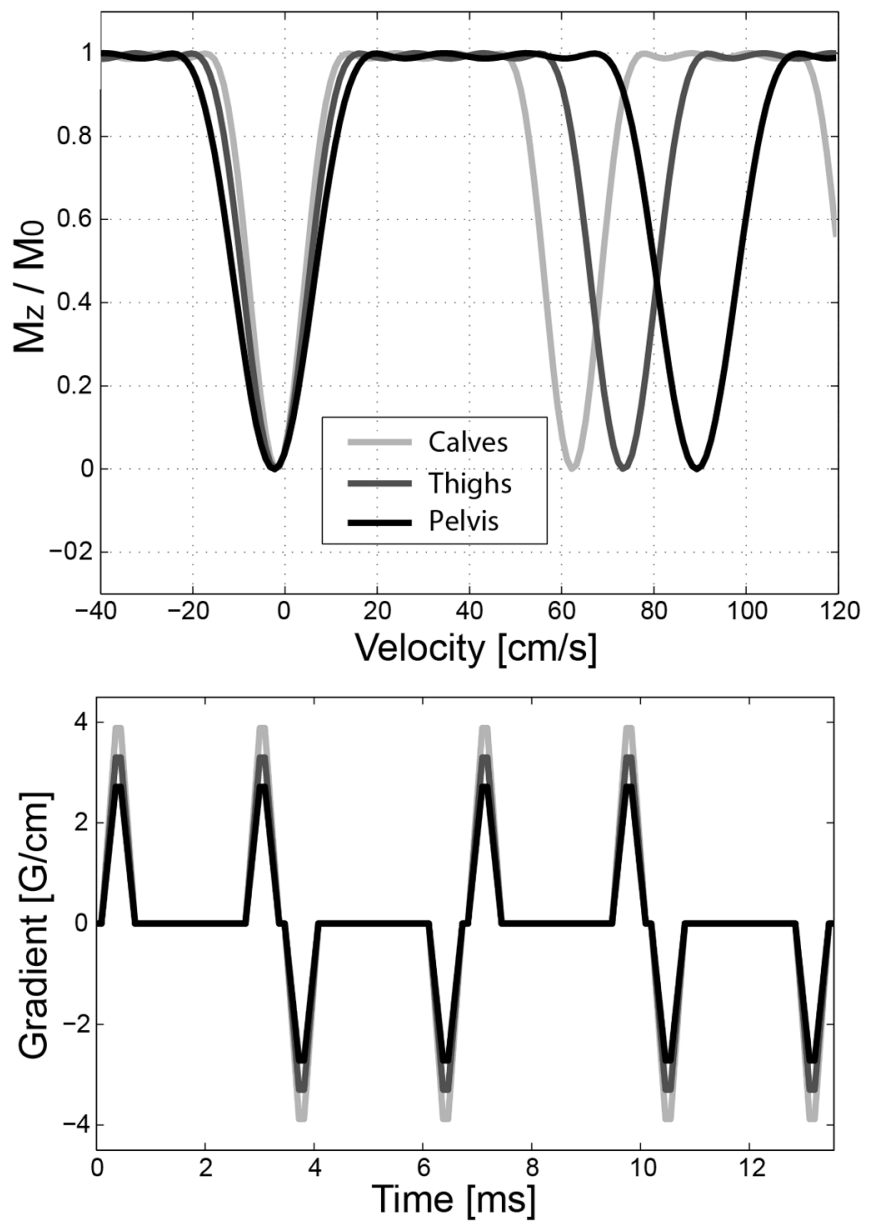

Figure 6.

Simulated $M_{\mathrm{Z}}$ profiles of VS saturation pulses (top) and corresponding VS gradient waveforms (bottom) for imaging the calves, thighs and pelvis. The baseline gradient waveform designed for the calves (lightest grey) was scaled down to increase the upper bound of the velocity pass-band for the thighs and pelvis which involve higher arterial flow. 


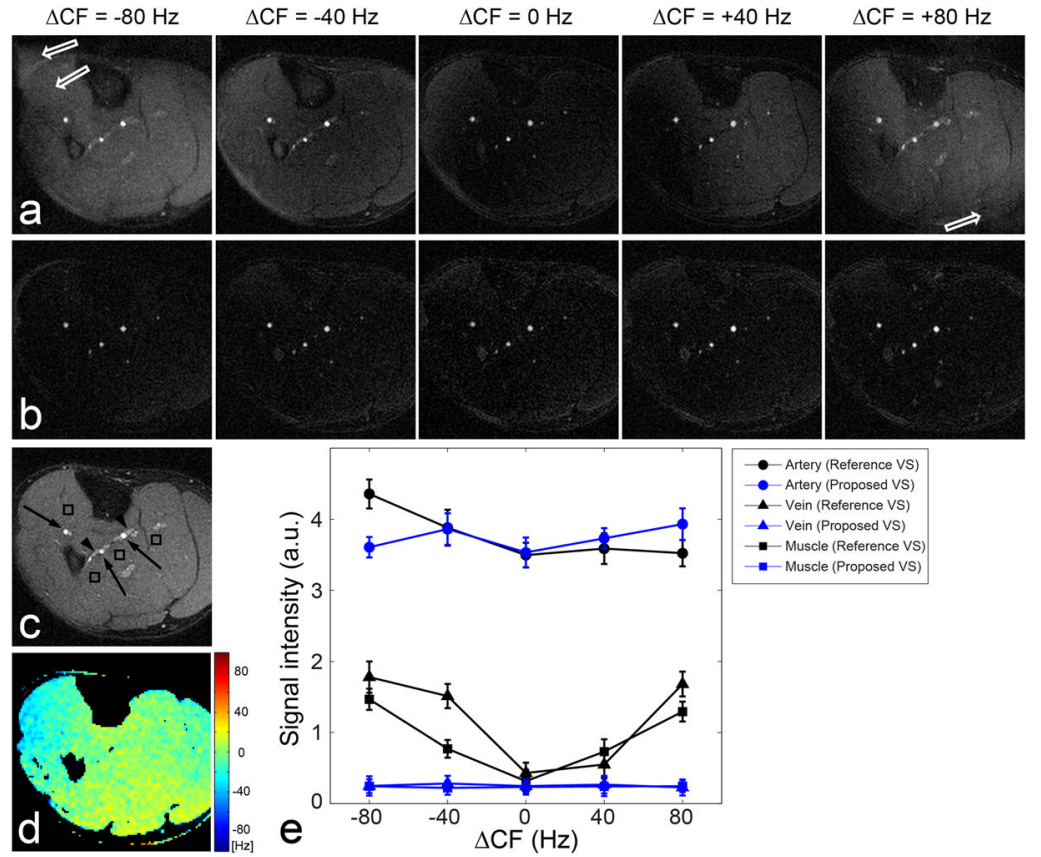

Figure 7.

Off-resonance sensitivity of VS saturation preparation for peripheral MRA in a subject. (a): Reference VS saturation yields excellent artery visualization and background suppression under a well-shimmed condition confirmed by the corresponding field map (d). However, background signal increases with increasing center frequency offset $(\triangle \mathrm{CF})$ due to the excitation profile shifting. The artery-to-background contrast achieved with $\triangle \mathrm{CF}= \pm 80 \mathrm{~Hz}$ appears to be similar to that of the reference image obtained without VS saturation preparation (c). (b): Proposed VS saturation (with refocusing and tailoring) maintains nearly the same artery-to-background contrast throughout all tested $\triangle \mathrm{CFs}$. ROI analysis of arteries, veins, and muscle (denoted by arrows, arrow heads, and rectangles, respectively, in c) across the center 24 slices demonstrate significantly improved off-resonance immunity of the proposed VS saturation compared to the reference VS saturation (e). 


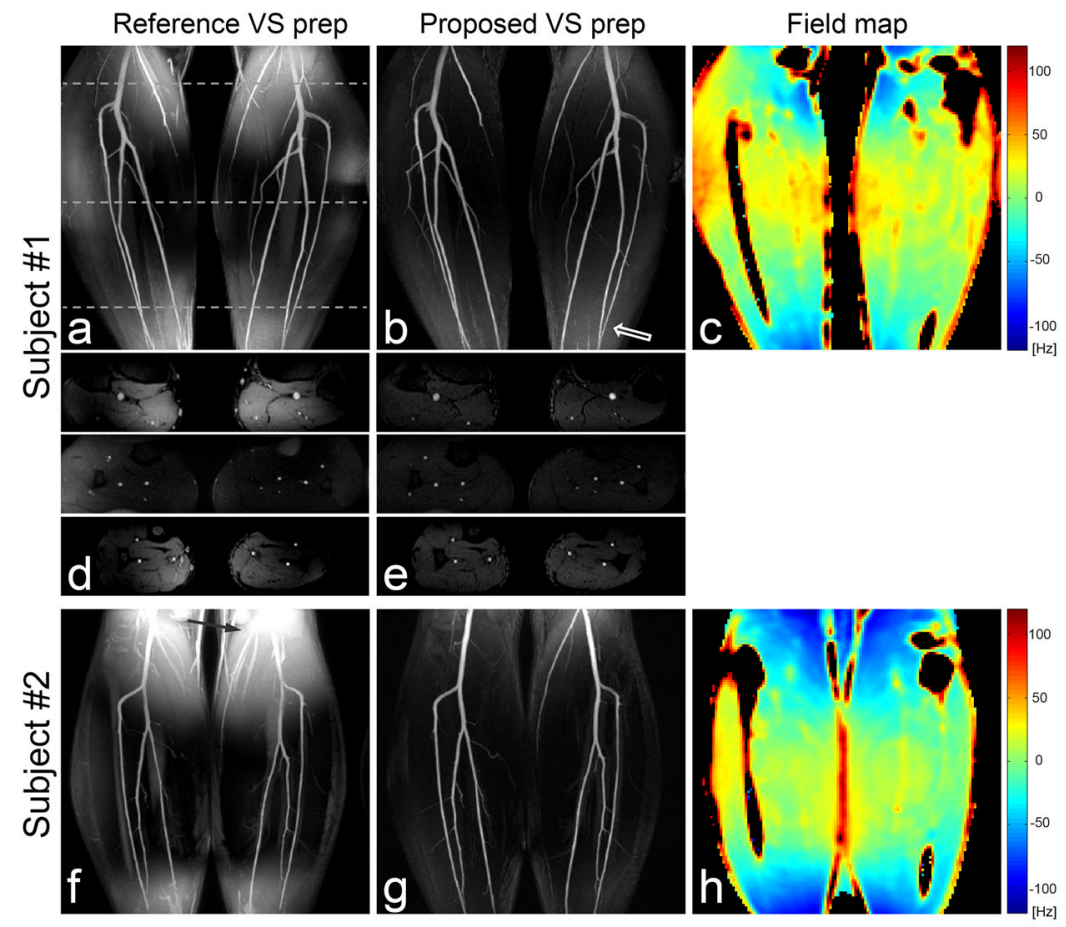

Figure 8.

Reformatted coronal maximum-intensity-projection (MIP) images of NCE MRA using the reference and proposed VS saturation preparations in the calves of two healthy subjects $(\mathrm{a}, \mathrm{b}, \mathrm{f}, \mathrm{g})$, and field maps of the center coronal planes $(\mathrm{c}, \mathrm{h})$. Representative axial slices at three S/I levels (denoted by dotted lines in a) are also shown for the first subject. MIP and axial images from the reference VS preparation suffer from poor background suppression in the region of large off-resonance. The off-resonance effect appears to be the largest near the top of the FOV in the $2^{\text {nd }}$ subject, consistent with the corresponding field map (solid arrow in $\mathrm{f}$ ). The proposed VS preparation yields excellent visualization of arteries and suppression of background tissues across the entire FOV due to its immunity to off-resonance. A slight increase in the background signal is observed in the regions with large superior/inferior offsets presumably due to $\mathrm{B}_{1}+$ variation (open arrow in $\mathrm{b}$ ). 

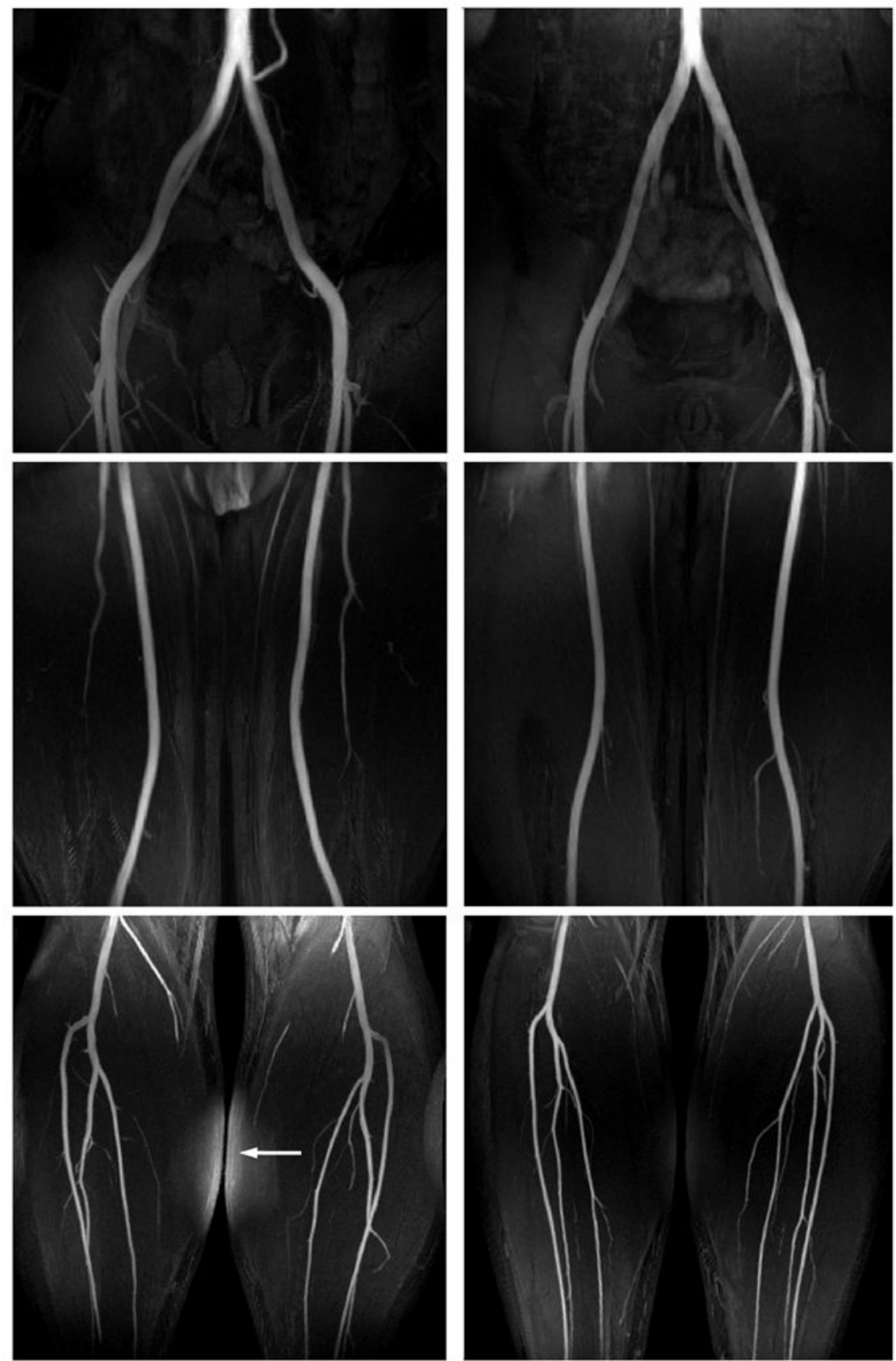

Figure 9.

Three-station NCE peripheral MRA using the proposed VS preparation in two healthy subjects. All major arteries are well visualized across a total of $90 \mathrm{~cm}-\mathrm{S} / \mathrm{I} \mathrm{FOV}(30 \mathrm{~cm}$ per station). Bright background signal in the calf station of the first subject is due to inadequate spectral coverage of the fat saturation pulse in the presence of large field inhomogeneity (arrow). 

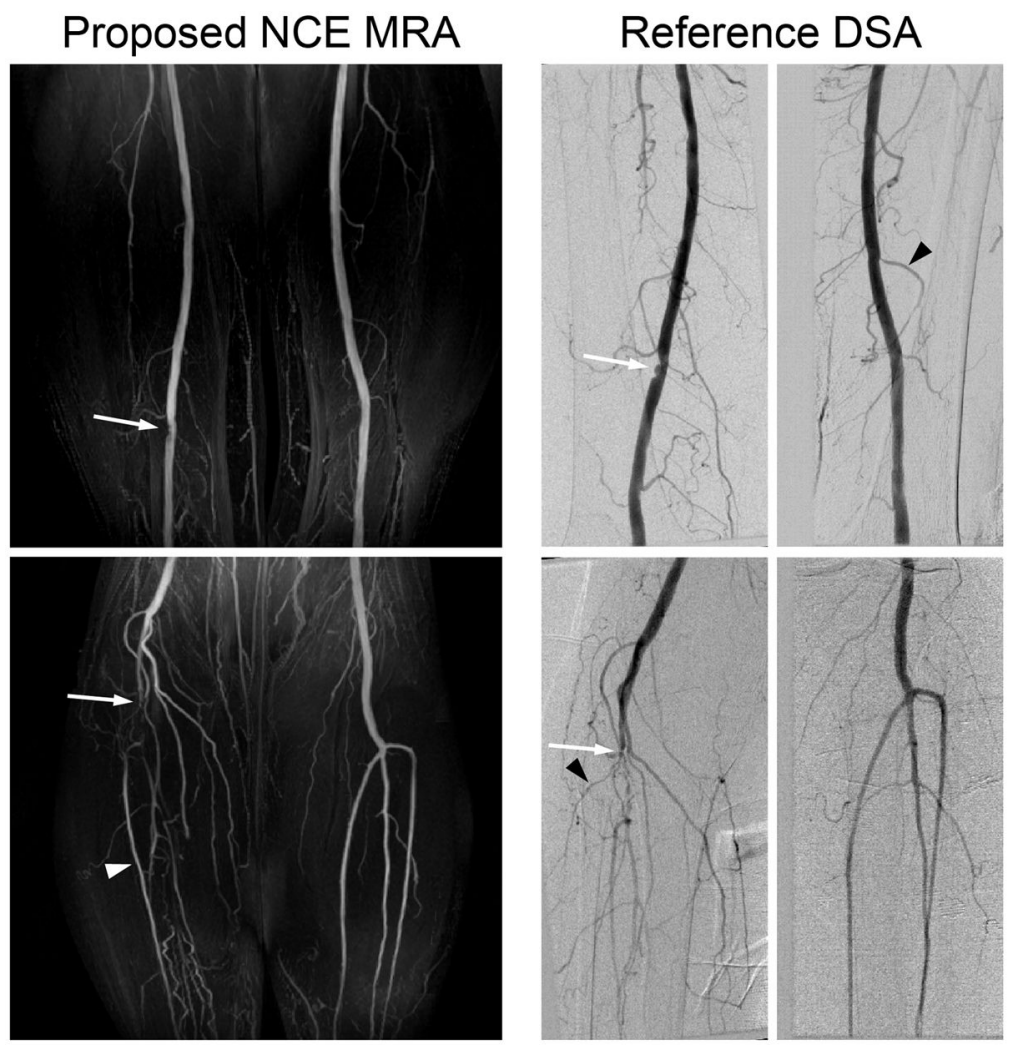

Figure 10.

Two-station NCE peripheral MRA in a 77-year-old female patient, compared with digital subtraction angiography (DSA). The MR angiograms agree well with the DSA images in identifying mild stenosis of the right femoral artery and occlusion of the right popliteal artery (arrows). Most segments of small vessels appear to be less clear in the MR images presumably due to lower spatial resolution and slow arterial flow (black arrowheads). The right anterior tibial artery is better visualized by NCE MRAas the concentration of X-ray contrast for DSA is expected to be limited in arteries distal to the occlusion (white arrowhead). 
